14

\title{
Параметры фазовой синхронизации в электроэнцефалографических паттернах как маркеры когнитивных нарушений
}

\author{
(C) О.Е. Дик, ${ }^{1}$, А.Л. Глазов ${ }^{2}$ \\ ${ }^{1}$ Институт ффизиологии им. И.П. Павлова РАН, \\ 199034 Санкт-Петербург, Россия \\ 2 Физико-технический институт им. А.Ф. Иофффе РАН, \\ 194021 Санкт-Петербург, Россия \\ e-mail: dickviola@gmail.com, glazov.holo@mail.ioffe.ru
}

Поступило в Редакцию 11 июня 2020 г.

В окончательной редакции 6 октября 2020 г.

Принято к публикации 26 октября 2020 г.

\begin{abstract}
Исследованы различия в фазовой синхронизации между прерывистой фотостимуляцией и биоэлектрической активностью мозга в виде электроэнцефалографических паттернов у двух групп лиц с хронически повышенным артериальным давлением, имеющих и не имеющих нарушения когнитивных функций. Показано, что параметры фазовой синхронизации, вычисленные на основе синхросжатого вейвлет-преобразования электроэнцефалографических паттернов и фотостимула, могут быть использованы в качестве нейрофизиологических маркеров умеренных когнитивных нарушений.
\end{abstract}

Ключевые слова: фазовая синхронизация, вейвлет-преобразование, электроэнцефалографические паттерны, артериальная гипертензия.

DOI: 10.21883/JTF.2021.04.50633.200-20

\section{Введение}

Анализ электроэнцефалографических (ЭЭГ) паттернов биоэлектрической активности мозга служит для диагностики функционального состояния нервной системы. Для оценки изменений этого состояния в клинической практике используется функциональная нагрузка в виде прерывистой фотостимуляции, поскольку иногда в фоновой ЭЭГ эти изменения обнаружить не удается [1]. Такая нагрузка применяется для определения возможности головного мозга воспроизводить или отклонять предложенный ритм [2,3]. Генерация мозгом ответа на частоте фотостимула или ее гармониках называется усвоением [4]. В зависимости от ответа на фотостимул определяется степень отклонения функционального состояния нервной системы от нормы [5].

При хроническом повышении артериального давления почти у $25 \%$ людей может возникать ухудшение когнитивных функций в виде нарушения кратковременной памяти и быстрой адаптации к изменяющимся обстоятельствам [6]. Известно, что при таком ухудшении в спектре мощности фоновой ЭЭГ наблюдается увеличение доли низкочастотных диапазонов [7]. Однако подобное увеличение отмечается, например, в фоновой ЭЭГ у пациентов, не имеющих когнитивных нарушений с диагнозом вертебробазилярной недостаточности $[3,8]$. Таким образом, для своевременной диагностики функционального состояния нервной системы у больных с таким социально значимым заболеванием, как артериальная гипертензия, при анализе динамики ЭЭГ важно анализировать изменения, происходящие не только в фоновых, но и в реактивных паттернах ЭЭГ.
Изменения в реактивных паттернах ЭЭГ под влиянием прерывистой фотостимуляции можно оценить на основе анализа вейвлетных спектров до и во время фотостимуляции. Такой анализ позволяет однозначно определить коэффициенты реакции усвоения и удержания ритма, а также время его запоминания по точкам пересечения кривых, соответствующих интегральным распределениям вейвлетных спектров ЭЭГ и светового стимула [3,9]. В этом случае интегральное распределение описывает долю энергии вейвлетного спектра в узком диапазоне частот вокруг частоты фотостимуляции. Следовательно, этот метод не предоставляет информацию о динамических изменениях, происходящих в паттернах ЭЭГ в случае появления в них частот, кратных частоте фотостимуляции. Это может быть сделано путем оценки изменений мгновенных частот и фаз паттернов ЭЭГ.

Одним из методов извлечения мгновенной фазы и частоты нестационарного сигнала является так называемый метод аналитического сигнала, включающий преобразование Гильберта $[10,11]$. Такой подход требует предварительной узкополосной фильтрации, поскольку в противном случае интерпретация мгновенной фазы, полученной после преобразования Гильберта, не имеет физического смысла [12]. Этот метод широко используется для выявления синхронизации между различными ритмами сердечно-сосудистой и дыхательной систем [13-15]. При этом R-R-интервалы основного сердечного ритма, кровяное давление и временные ряды дыхания исследуются в определенных узких диапазонах $0.04-0.15 \mathrm{~Hz}$ или $0.15-0.4 \mathrm{~Hz}$. Временна́я последовательность ЭЭГ включает в себя более широкий диапа- 
зон, поэтому применение такого подхода для извлечения мгновенных частот и фаз будет некорректным.

Альтернативой методу аналитического сигнала является метод расчета мгновенных фаз и частот на основе вейвлет-преобразования сигнала [16]. Этот метод применяется при изучении синхронизации колебаний основного сердечного ритма и ритма дыхания $[17,18]$. Вейвлетный анализ паттернов ЭЭГ позволяет оценить динамическую сложность физиологических реакций в зависимости от степени патологии $[19,20]$. Для повышения эффективности извлечения мгновенной частоты и фазы из экспериментальных данных с высоким уровнем шума применяется метод синхросжатого вейвлетпреобразования [21].

Целью настоящей работы является оценка параметров фазовой синхронизации между прерывистой фотостимуляцией и реакцией мозга в виде его биоэлектрической активности при артериальной гипертензии на основе синхросжатого вейвлет-преобразования.

\section{1. Методы}

\section{1. Экспериментальные данные}

В настоящей работе проанализированы записи ЭЭГ 30 пациентов (женщин) с диагнозом артериальной гипертензии второй стадии (повышенное систолическое артериальное давление в диапазоне $160-180 \mathrm{~mm} \mathrm{Hg}$ и диастолическое в диапазоне $100-110 \mathrm{~mm} \mathrm{Hg}$ ). Данные были предоставлены Санкт-Петербургской неврологической клиникой, исследование было одобрено местным комитетом по этике. По данным МРТ отмечались умеренные нарушения функционального состояния мозга, соответствующие начальной стадии сосудистой энцефалопатии (нарушения мозгового кровообращения, связанного с хроническим повышением артериального давления). Среди этих пациентов 13 человек (группа I, средний возраст $63.3 \pm 7.7$ года) имели начальные проявления умеренных когнитивных расстройств. У 17 пациентов группы II (средний возраст $61.5 \pm 3.1$ года) когнитивные расстройства отсутствовали. Умеренные когнитивные расстройства проявлялись в виде неустойчивости умственной работоспособности и снижения уровня психической активности, но без дезадаптаций в повседневной жизни и ограничения самостоятельности. В анамнезе пациентов всех групп отсутствовали инсульты и инфаркты, признаки избыточного скопления цереброспинальной жидкости в желудочковой системе головного мозга (гидроцефалии), болезни Альцгеймера и Паркинсона также отсутствовали. Наряду с когнитивными расстройствами, практически у всех больных отмечалась ишемическая болезнь сердца. Контрольная группа здоровых добровольцев состояла из 10 человек (средний возраст $31.5 \pm 7.7$ года), имеющих нормальное артериальное давление $(80 / 120 \mathrm{~mm} \mathrm{Hg})$.
Данные содержали безартефактные паттерны ЭЭГ до, во время и после прерывистой фотостимуляции. Фотостимуляция представляла собой последовательность восьмисекундных серий световых импульсов с разной частотой с интервалом $30 \mathrm{~s}$ между сериями. Данные ЭЭГ представляли собой ответы мозга в затылочных локуcax $\mathrm{O} 1, \mathrm{O} 2, \mathrm{Oz}$, где реакции мозга на световой стимул имеют наибольшую амплитуду, в диапазоне $1-25 \mathrm{~Hz}$.

\section{2. Оценка фазовой синхронизации}

Появление фазовой синхронизации между паттерном ЭЭГ и фотостимулом на заданной частоте оценивали методом синхросжатого вейвлет-преобразования [21].

Непрерывное вейвлет-преобразование (CWT) временного ряда $s(t)$ для вейвлетной функции $\psi(t)$ записывается в виде [16]:

$$
W_{s}(a, b)=\frac{1}{a} \int_{-\infty}^{+\infty} s(t) \bar{\psi}\left(\frac{t-b}{a}\right) d t,
$$

где $a$ и $b$ - переменные масштаба и временного сдвига, $\psi((t-b) / a)$ - вейвлетная функция, полученная из материнского вейвлета $\psi(t)$ путем масштабирования и сдвига по времени, символ $\bar{\psi}$ означает комплексное сопряжение. Это преобразование может быть использовано для определения мгновенной частоты сигнала [21]:

$$
\omega_{s}(a, b)=\operatorname{Im}\left(\frac{1}{W_{s}(a, t)} \frac{\partial W_{s}(a, b)}{\partial b}\right),
$$

где круговая частота $\omega_{s}(a, b)$ сигнала $s(t)$ связана с масштабом $a=a(b)$ и центральной частотой вейвлета $\omega_{0}$ соотношением

$$
\omega_{s}(a, b)=\omega_{0} / a(b) .
$$

Интерпретация результирующего спектрального разложения часто затрудняется размытием некоторых частотных составляющих анализируемого сигнала [22]. Уменьшить эти эффекты можно с использованием метода синхросжатого вейвлет-преобразования [23].

Синхросжатое вейвлет-преобразование (SWT) сигнала $s(t)$ вычисляется в центрах $\omega_{l}$ последовательных интервалов

$$
\begin{gathered}
{\left[\omega_{l}-\Delta \omega / 2, \omega_{l}+\Delta \omega / 2\right], \quad \omega_{l}=2 \pi(l / n) F_{S},} \\
\Delta \omega=F_{S} / n, \quad l=1, \ldots, n,
\end{gathered}
$$

где $F_{S}$ - частота дискретизации сигнала, $n-$ число масштабов, используемых при построении вейвлетного спектра $T_{s}\left(\omega_{l}, b\right)$.

Метод извлечения гребня (временной последовательности максимумов вейвлет-коэффициентов) из SWTсигнала сводится к решению задачи условной оптимизации поиска среди всех кривых $\omega_{r}(b)=\arg \max \left|T_{s}\left(\omega_{l}, b\right)\right|$ 

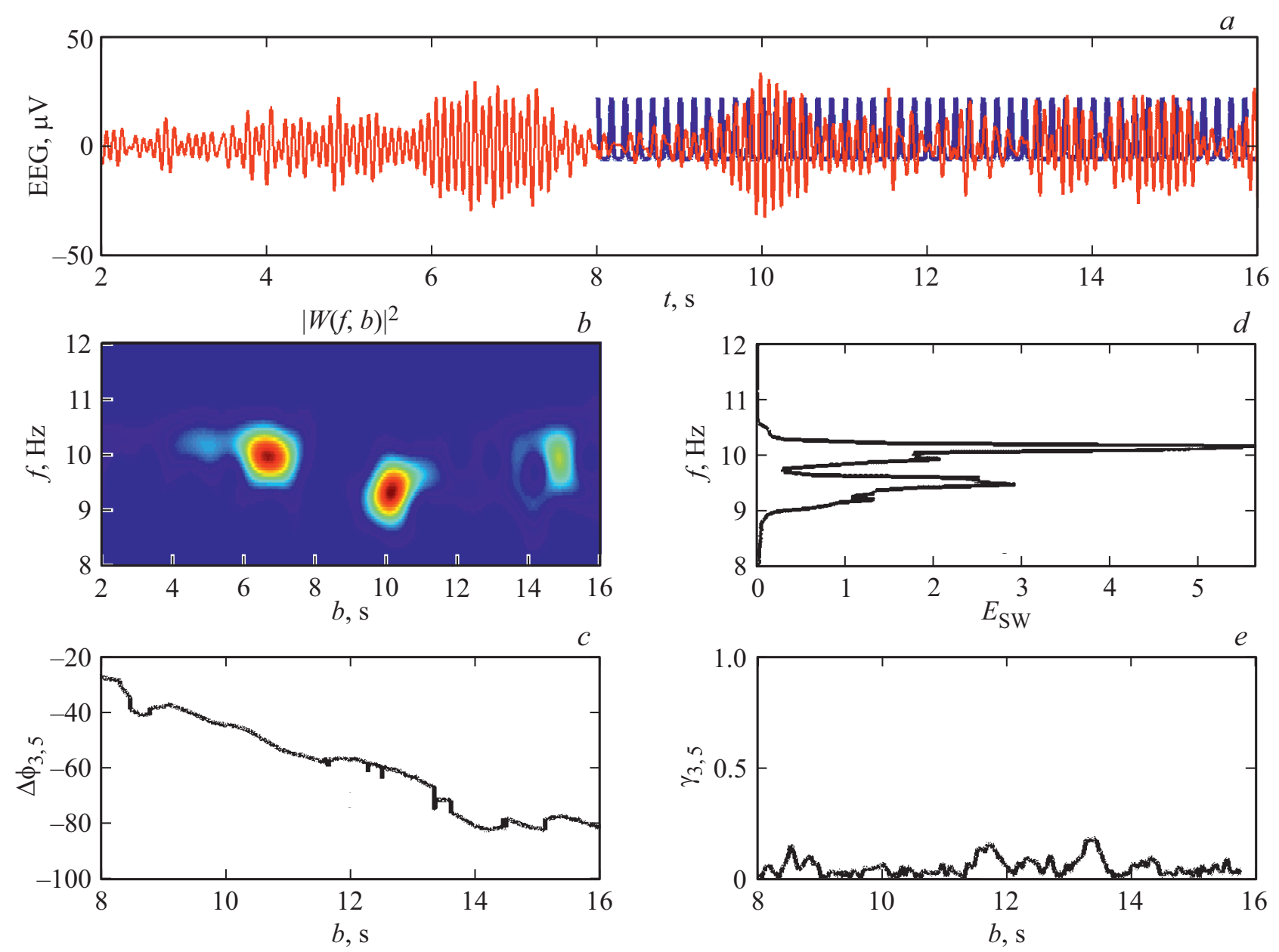

Рис. 1. Реакция мозга здорового человека на фотостимуляцию с частотой $6 \mathrm{~Hz} . a-$ паттерн ЭЭГ (красная кривая в оnline версии) и фотостимул (голубая кривая в оnline версии), $b$ - проекция вейвлетной поверхности $\left(b, f,\left|W_{s}(f, b)\right|^{2}\right)$ на плоскость $(b, f)$, полученная на основе CWT паттерна ЭЭГ, $c-$ зависимость мгновенной разности фаз от времени $\Delta \phi_{3,5}(b), d-$ усредненное по времени распределение вейвлетного спектра $\left|W_{s}(f, b)\right|^{2}$ по частотам, $e-$ зависимость индекса фазовой синхронизации $\gamma_{3,5}$ от времени.

той, которая максимизирует модуль $T_{s}\left(\omega_{l}, b\right)$ вдоль гребня [24].

На основании полученных гребней $\omega_{r}(b)$ мгновенная частота $f_{s}(b)$ и мгновенная фаза $\phi_{s}(b)$ сигнала определяются по формулам реконструкции гребня [23]:

$$
f_{s}(b)=\omega_{r}(b) / 2 \pi, \quad \phi_{s}(b)=\arg \left|T_{s}\left(\omega_{r}(b), b\right)\right| .
$$

Если в анализируемом временном интервале обнаруживается несколько гребней, то процедуру извлечения гребней можно повторить.

В качестве вейвлетной функции в работе использован вейвлет Габора-Морле

$$
\begin{aligned}
\psi((t-b) / a)= & (1 / a) \exp \left[i \omega_{0}((t-b) / a)\right] \\
& \times \exp \left(-0.5((t-b) / a)^{2}\right)
\end{aligned}
$$

при значении параметра $\omega_{0}=2 \pi f_{0}, f_{0}=1$, что обеспечивает простую связь между масштабом а вейвлетпреобразования и реальной частотой анализируемого сигнала [25]

$$
f=\left(\left(\omega_{0}+\sqrt{2+\omega_{0}^{2}}\right) / 4 \pi a\right) \approx 1 / a .
$$

Интеграл

$$
E_{S W}(f)=\int_{t_{1}}^{t_{2}}\left|T_{S}(f, b)\right|^{2} d b
$$

определяет усредненное по времени распределение энергии синхросжатого вейвлетного спектра по частотам на заданном временном интервале $\left[t_{1}, t_{2}\right]$.

Световой стимул $p(t)$ аппроксимировался последовательностью $k$ гауссовых импульсов, следующих друг за другом с частотой $f_{p}$. Каждый импульс имел ширину $r_{0}=10 \mathrm{~ms}$, а центры импульсов были расположены в точках $t_{j}=t_{A}+j / f_{p}, j=0, \ldots, k-1$, где $t_{A}-$ время начала первого импульса в последовательности

$$
p(t)=\sum_{j=0}^{k-1} \frac{0.5}{r_{0} \sqrt{\pi}} \exp \left(-\frac{\left(t-t_{j}\right)^{2}}{4 r_{0}^{2}}\right) .
$$



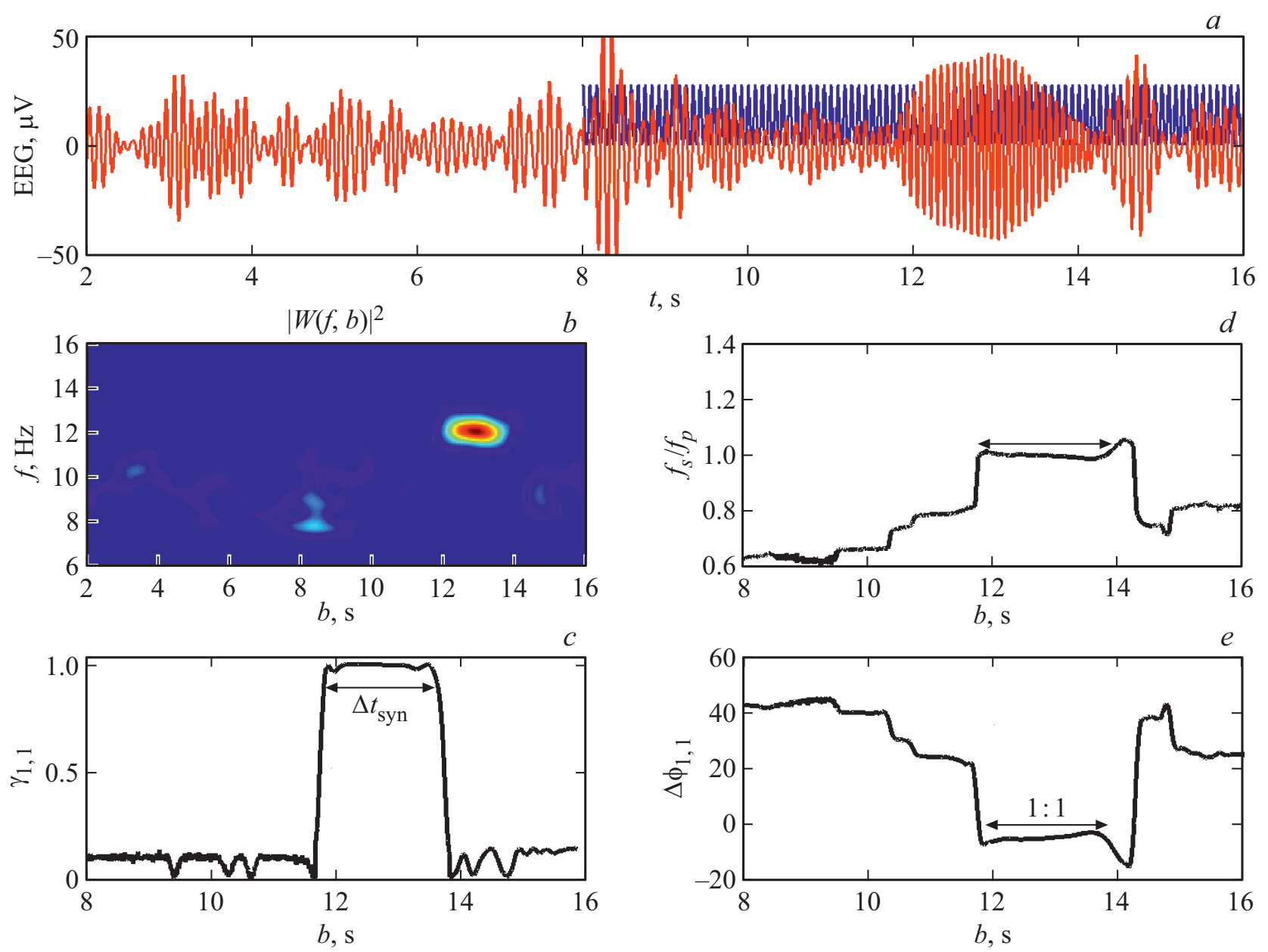

Рис. 2. Пример фазовой синхронизации между световым возбуждением на частоте $12 \mathrm{~Hz}$ и ответом здорового мозга. $a-$ паттерн ЭЭГ (красная кривая в online версии) и фотостимул (голубая кривая в online версии), $b$ - проекция вейвлетной поверхности $\left(b, f,\left|W_{s}(f, b)\right|^{2}\right)$ на плоскость $(b, f), c-$ зависимость индекса фазовой синхронизации $\gamma_{1,1}$ от времени, $d-$ отношение мгновенных частот $f_{s}(b) / f_{p}(b), e-$ мгновенная разность фаз $\Delta \phi_{1,1}(b)$.

Применяя SWT к сигналам $s(t)$ и $p(t)$ и оценивая их мгновенные частоты и фазы, мы вычислили соотношение мгновенных частот $f_{s}(b) / f_{p}(b)$ и безразмерную разность мгновенных фаз

$$
\Delta \phi_{n, m}(b)=\left(n \phi_{s}(b)-m \phi_{p}(b)\right) / 2 \pi,
$$

где $n$ и $m$ - целые числа.

Наличие фазовой синхронизации $n: m$ между паттерном ЭЭГ и фотостимулом данной частоты определялось условием [26]:

$$
\left|\Delta \phi_{n, m}(b)-c_{1}\right|<\varepsilon_{1},
$$

где $c_{1}-$ константа и $\varepsilon_{1}=0.03$. Это означает, что в случае синхронизации фаз $n: m$ между двумя сигналами мгновенная разность фаз $\Delta \phi_{n, m}(b)$ колеблется вокруг постоянного значения $c_{1}$.

Отношение мгновенных частот $f_{s}(b) / f_{p}(b)$ в случае синхронизации колеблется вокруг постоянного значения $c_{2}=m / n$, так что выполняется

$$
\left|f_{s}(b) / f_{p}(b)-c_{2}\right|<\varepsilon_{2},
$$

где $\varepsilon_{2}=0.03$.

Длительность фазовой синхронизации $n: m$ между световым возбуждением и реакцией мозга на заданную частоту определялась как интервал времени $\Delta t_{\mathrm{syn}}$, в течение которого значение индекса фазовой синронизации, вычисляемое в соответствии с [27],

$$
\begin{aligned}
\gamma_{n, m} & =\left|\left\langle\exp \left(2 \pi i \Delta \phi_{n, m}(b)\right)\right\rangle_{[b+\Delta b]}\right| \\
& =\left|\sum_{j=1}^{k} \exp \left(2 \pi i \Delta \phi_{n, m}\left(b+\frac{j \Delta b}{k}\right)\right)\right|
\end{aligned}
$$

было близко к единице.

Таким образом, фазовая синхронизация между паттерном ЭЭГ и фотостимулом заданной частоты оценивалась в настоящей работе по таким параметрам, как отношение мгновенных частот и длительность фазовой синхронизации.

Различия между средними значениями вычисленных параметров для каждой группы пациентов и средними 

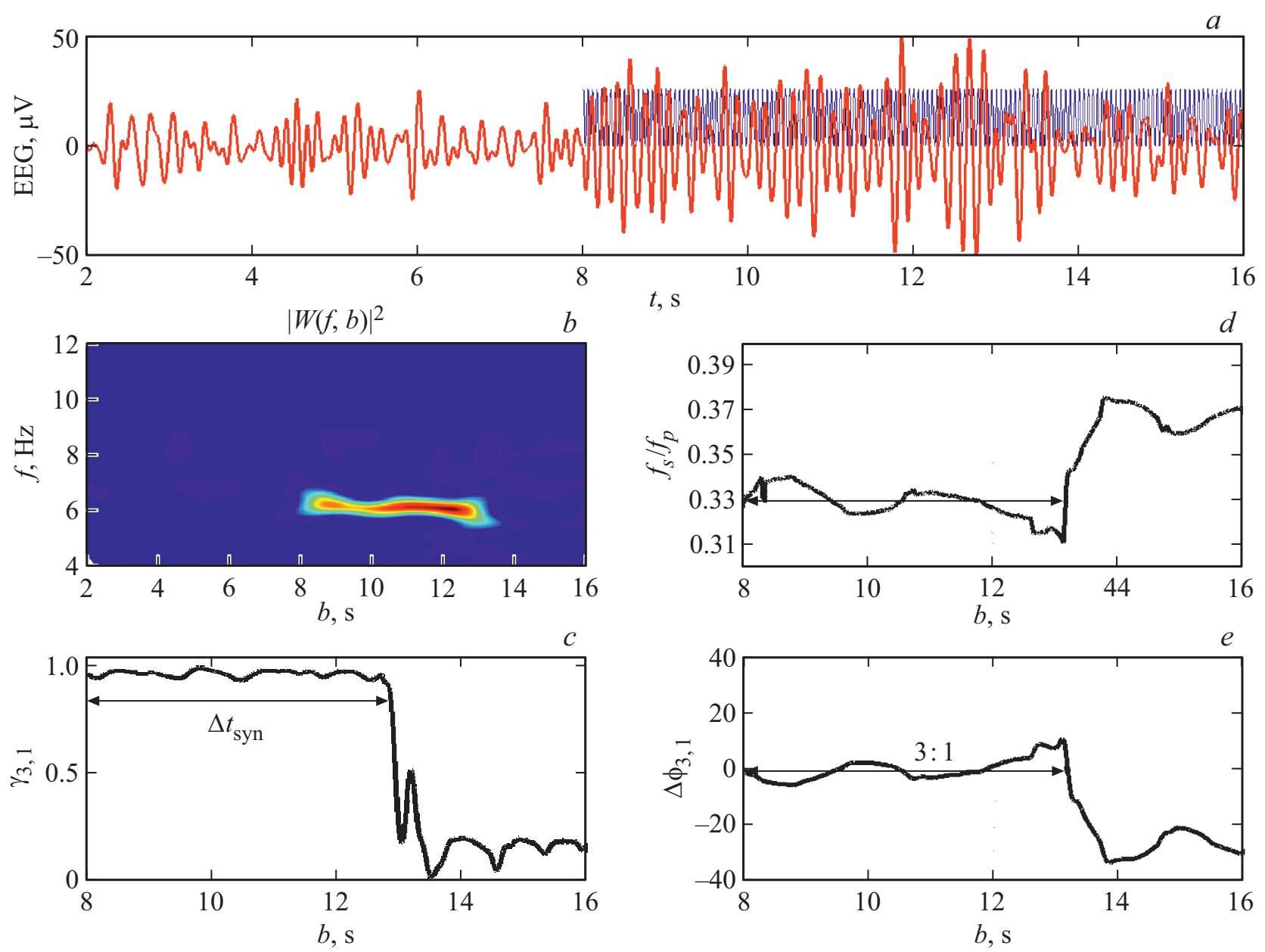

Рис. 3. Пример фазовой синхронизации порядка $3: 1$ между световым возбуждением с частотой 18 Нz и ответом мозга человека из группы I. $a$ - паттерн ЭЭГ (красная кривая в online версии) и фотостимул (голубая кривая в оnline версии), $b-$ проекция вейвлетной поверхности $\left(b, f,\left|W_{s}(f, b)\right|^{2}\right)$ на плоскость $(b, f), c$ - зависимость индекса фазовой синхронизации $\gamma_{3,1}$ от времени, $d$ - отношение мгновенных частот $f_{s}(b) / f_{p}(b), e-$ мгновенная разность фаз $\Delta \phi_{3,1}(b)$.

параметрами контрольной группы, а также между этими средними значениями для группы I и группы II пациентов определяли по методу однофакторного дисперсионного анализа ANOVA. Если статистика, полученная по Fкритерию Фишера, превышала критическое значение, то нулевая гипотеза о равенстве средних двух групп отвергалась.

\section{2. Результаты}

В связи с тем. что значения параметров, вычисленных для всех трех затылочных локусов, значимо не различались на статистическом уровне, в настоящей работе приводятся результаты, полученные только для центрального затылочного локуса $\mathrm{Oz}$.

Реакция мозга здорового человека из контрольной группы на фотостимуляцию с частотой $6 \mathrm{~Hz}$ показана на рис. 1. Действие фотостимула длилось с восьмой по шестнадцатую секунду (рис. 1,a). Проекция вейвлетного спектра $\left(b, f,\left|W_{s}(f, b)\right|^{2}\right)$ на плоскость $(b, f)$, полученная в результате CWT ЭЭГ до и во время фотостимуляции, показана на рис. $1, b$. Усредненное по времени распределение вейвлетного спектра $\left|W_{s}(f, b)\right|^{2}$ по частотам имеет максимумы на частотах 9.5 и $10 \mathrm{~Hz}$, не совпадающих с частотой фотостимуляции (рис. $1, d)$. Зависимость мгновенной разности фаз от времени $\Delta \phi_{3,5}(b)$ не имеет участков горизонтального плато (рис. 1,c). Индекс фазовой синхронизации $\gamma_{3,5}$ колеблется вокруг значения, близкого к нулю (рис. $1, e$ ). Все это свидетельствует об отсутствии фазовой синхронизации и реакции усвоения светового ритма заданной частоты $6 \mathrm{~Hz}$ в анализируемом паттерне ЭЭГ с максимумом мгновенной частоты $10 \mathrm{~Hz}$.

На рис. 2 приведен пример наличия фазовой синхронизации между световым возбуждением на частоте $12 \mathrm{~Hz}$ и реакцией здорового мозга. Проекция вейвлетного спектра $\left(b, f,\left|W_{s}(f, b)\right|^{2}\right)$ паттерна ЭЭГ на плоскость $(b, f)$ показывает отсутствие в спектре частоты $12 \mathrm{~Hz}$ до фотостимуляции и появление ее во время фотостимуляции (рис. 2, $b$ ). Отношение мгно- 

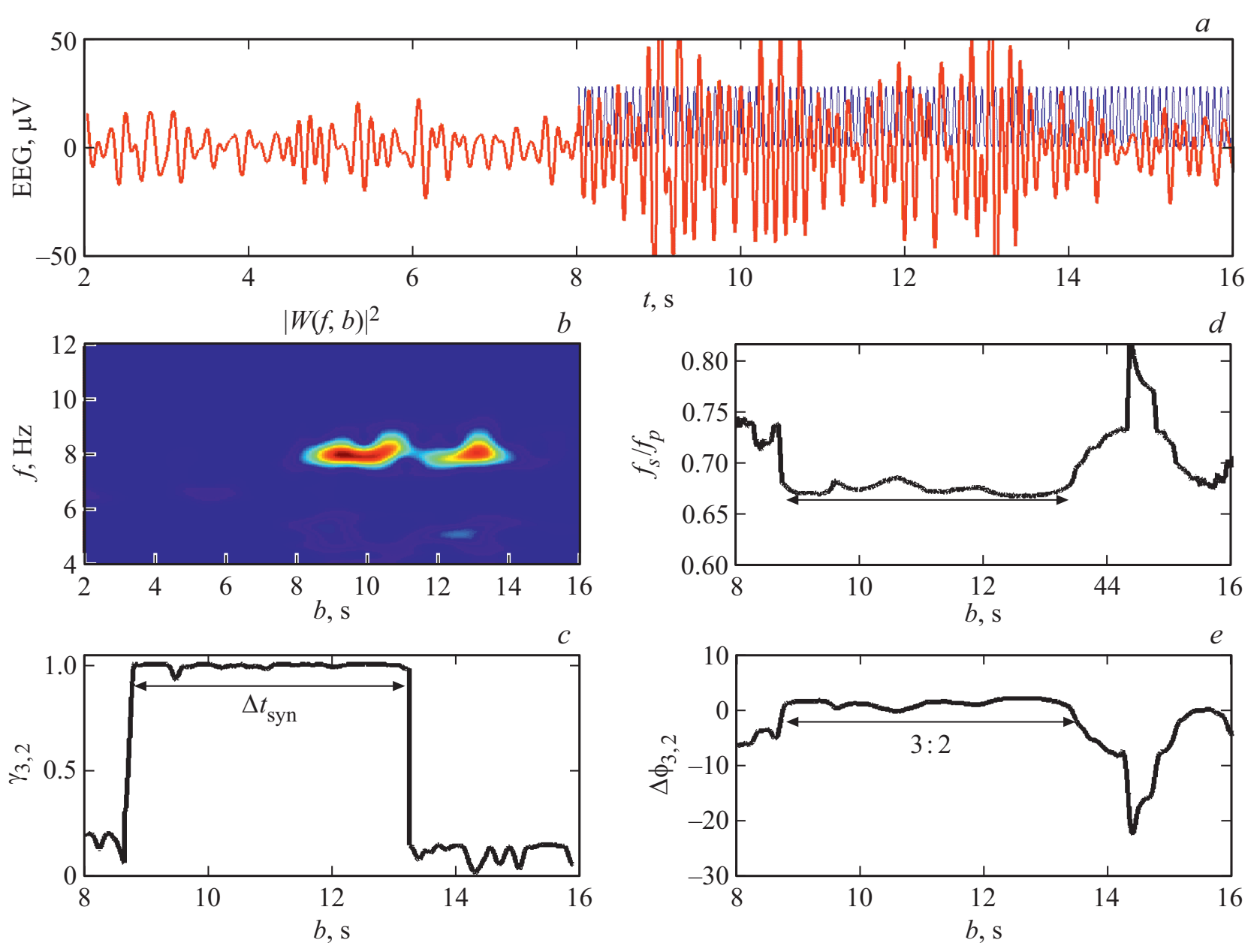

Рис. 4. Пример фазовой синхронизации порядка $3: 2$ между световым возбуждением с частотой 12 Нz и ответом мозга человека из группы I. $a$ - паттерн ЭЭГ (красная кривая в online версии) и фотостимул (голубая кривая в оnline версии ), $b-$ проекция вейвлетной поверхности $\left(b, f,\left|W_{s}(f, b)\right|^{2}\right)$ на плоскость $(b, f), c$ - зависимость индекса фазовой синхронизации $\gamma_{3,2}$ от времени, $d$ - отношение мгновенных частот $f_{s}(b) / f_{p}(b), e-$ мгновенная разность фаз $\Delta \phi_{3,2}(b)$.

венных частот $f_{s}(b) / f_{p}(b)$ ответа мозга и светового возбуждения колеблется вокруг постоянного значения, близкого к единице (рис. 2,d), а мгновенная разность фаз $\Delta \phi_{1,1}(b)$ - вокруг постоянного значения, близкого к нулю в интервале времени от 11.8 до $13.9 \mathrm{~s}$ (рис. 2,e). Это свидетельствует о том, что спустя $3.8 \mathrm{~s}$ после начала фотостимуляции между световым возбуждением и реакцией здорового мозга наступает фазовая синхронизация порядка $1: 1$. Индекс фазовой синхронизации $\gamma_{1,1}$ имеет значения, близкие к единице внутри того же самого интервала, и принимает значения, близкие к нулю, вне этого интервала (рис. 2, c). Таким образом, длительность $\Delta t_{\text {syn }}$ фазовой синхронизации в этом примере составляет $2.2 \mathrm{~s}$.

Пример наличия фазовой синхронизации порядка $3: 1$ между световым возбуждением с частотой $18 \mathrm{~Hz}$ и ответом мозга человека с артериальной гипертензией из группы I показан на рис. 3. Вейвлетный спектр $\left|W_{s}(f, b)\right|^{2}$ ЭЭГ выявляет частоту ответа $6 \mathrm{~Hz}$ в интервале времени от 8 до $13 \mathrm{~s}$ (рис. $3, b$ ). В этом интервале времени отношение мгновенных частот $f_{s}(b) / f_{p}(b)$ колеблется вокруг постоянного значения, близкого к 0.33 (рис. $3, d$ ), а мгновенная разность фаз $\Delta \phi_{3,1}(t)-$ вокруг постоянного значения, близкого к нулю (рис. $3, e$ ). Внутри этого интервала индекс фазовой синхронизации $\gamma_{3,1}$ имеет значения, близкие к единице (рис. 3,c). Длительность $\Delta t_{\text {syn }}$ фазовой синхронизации в рассмотренном паттерне ЭЭГ равна $5 \mathrm{~s}$.

Рис. 4 демонстрирует фазовую синхронизацию порядка 3:2 между фотостимулом с частотой $12 \mathrm{~Hz}$ и ответом мозга человека с артериальной гипертензией из группы I. В вейвлетном спектре $\left|W_{s}(f, b)\right|^{2}$ этого паттерна ЭЭГ во время фотостимуляции появляется частота $8 \mathrm{~Hz}$ в интервале времени от 8.7 до $13.4 \mathrm{~s}$ (рис. $4, b$ ). В этом интервале значение индекса фазовой синхронизации $\gamma_{3,2}$ близко к единице (рис. 4,c), отношение мгновенных частот $f_{s}(b) / f_{p}(b)$ колеблется вокруг постоянного значения, близкого к 0.67 (рис. 4, $d$ ), а мгновенная разность фаз $\Delta \phi_{3,2}(b)-$ вокруг постоянного значения, близкого к нулю (рис. $4, e)$. Длительность $\Delta t_{\mathrm{syn}}$ 

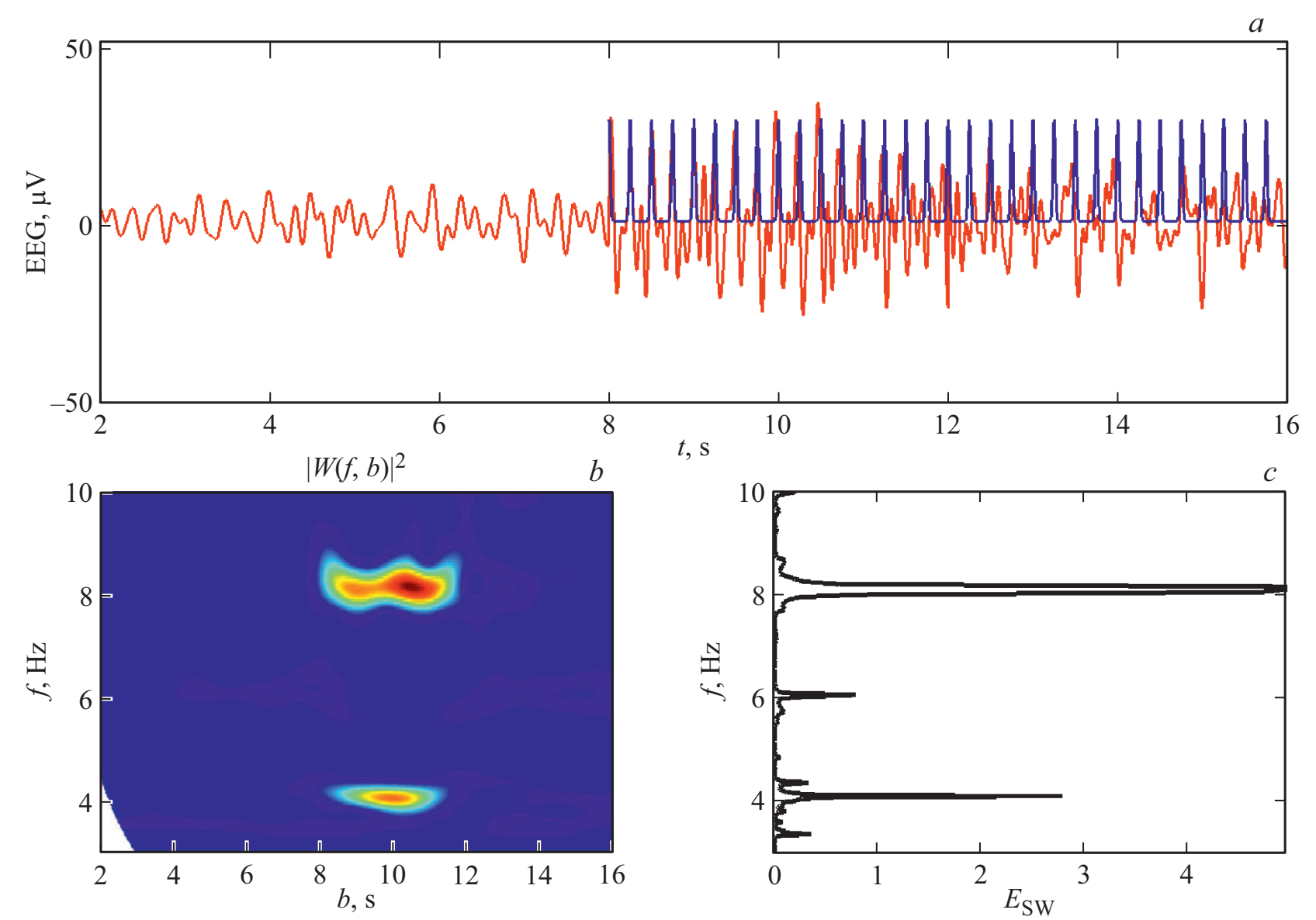

Рис. 5. Пример наличия двух порядков $n: m$ фазовой синхронизации между фотостимулом с частотой $4 \mathrm{~Hz}$ и реакцией мозга человека из группы II. $a$ - паттерн ЭЭГ (красная кривая в online версии) и фотостимул (голубая кривая в оnline версии), $b-$ проекция вейвлетной поверхности $\left(b, f,\left|W_{s}(f, b)\right|^{2}\right)$ на плоскость $(b, f), c$ - усредненное по времени распределение вейвлетного спектра $\left|W_{s}(f, b)\right|^{2}$ по частотам.

фазовой синхронизации между световым возбуждением и ответом в данном паттерне ЭЭГ составляет $4.7 \mathrm{~s}$.

Рис. 5 и 6 демонстрируют наличие двух порядков $\mathrm{n}: \mathrm{m}$ фазовой синхронизации между фотостимулом с частотой $4 \mathrm{~Hz}$ и реакцией мозга человека с артериальной гипертензией из группы II. Вейвлетный спектр $\left|W_{s}(f, b)\right|^{2}$ паттерна ЭЭГ имеет две полосы частот, возникающих в интервале фотостимуляции (рис. $5, b$ ). До фотостимуляции эти частоты отсутствуют. SWT выделяет два гребня, соответствующих максимумам усредненного по времени вейвлетного спектра $\left|T_{s}(f, b)\right|^{2}$ на частотах 4 и $8 \mathrm{~Hz}$ (рис. $5, c)$.

Фазовая синхронизация порядков $1: 1$ и $1: 2$ возникает в интервале времени от 8 до $11.8 \mathrm{~s}$ (рис. 6). В этом интервале времени отношение мгновенных частот $f_{s}(b) / f_{p}(b)$ колеблется вокруг постоянных значений, близких к 2 (рис. $6, a$ ) и к единице (рис. $6, d$ ), а мгновенные разности фаз $\Delta \phi_{1,2}(b)$ и $\Delta \phi_{1,1}(b)-$ вокруг постоянного значения, близкого к нулю (рис. $6, b, e$ ). Значения индексов фазовой синхронизации $\gamma_{1,2}$ и $\gamma_{1,1}$ близки к единице (рис. $6, c, f)$. Таким образом, длительность $\Delta t_{\text {syn }}$ фазовой синхронизации между световым возбуж- дением и ответом в данном паттерне ЭЭГ меньше, чем в паттернах, представленных на рис. 3 и 4.

В табл. 1 и 2 представлены данные о порядках $\mathrm{n}: \mathrm{m}$ фазовой синхронизации между фотостимулом и реакцией мозга пациентов с артериальной гипертензией из групп I и II соответственно. Для $61 \%$ (8 из 13) пациентов из группы I характерна фазовая синхронизация порядка $1: 1$ для фотостимула с частотами 4,6 и $10 \mathrm{~Hz}$, а также фазовая синхронизация порядка $2: 1$ для частот 10 и $12 \mathrm{~Hz}$, порядка $3: 2$ для частоты $12 \mathrm{~Hz}$ и порядков $4: 1$ и $3: 1$ для 16 и $18 \mathrm{~Hz}$ соответственно.

Для 59\% (10 из 17) человек из группы II в паттернах ЭЭГ найдена фазовая синхронизация порядка $1: 1$ между ответом мозга и световым возбуждением с частотами 4 , 6,10 и $12 \mathrm{~Hz}$ и фазовая синхронизация порядка $1: 2$ для фотостимула с частотами 4 и $6 \mathrm{~Hz}$. В некоторых паттернах ЭЭГ пациентов этой группы наблюдалась фазовая синхронизация порядков 4:5 и 4:7 между частотой возбуждения $4 \mathrm{~Hz}$ и ответом мозга и фазовая синхронизация порядка $1: 3$ между фотостимулом с частотой $6 \mathrm{~Hz}$ и ЭЭГ. 

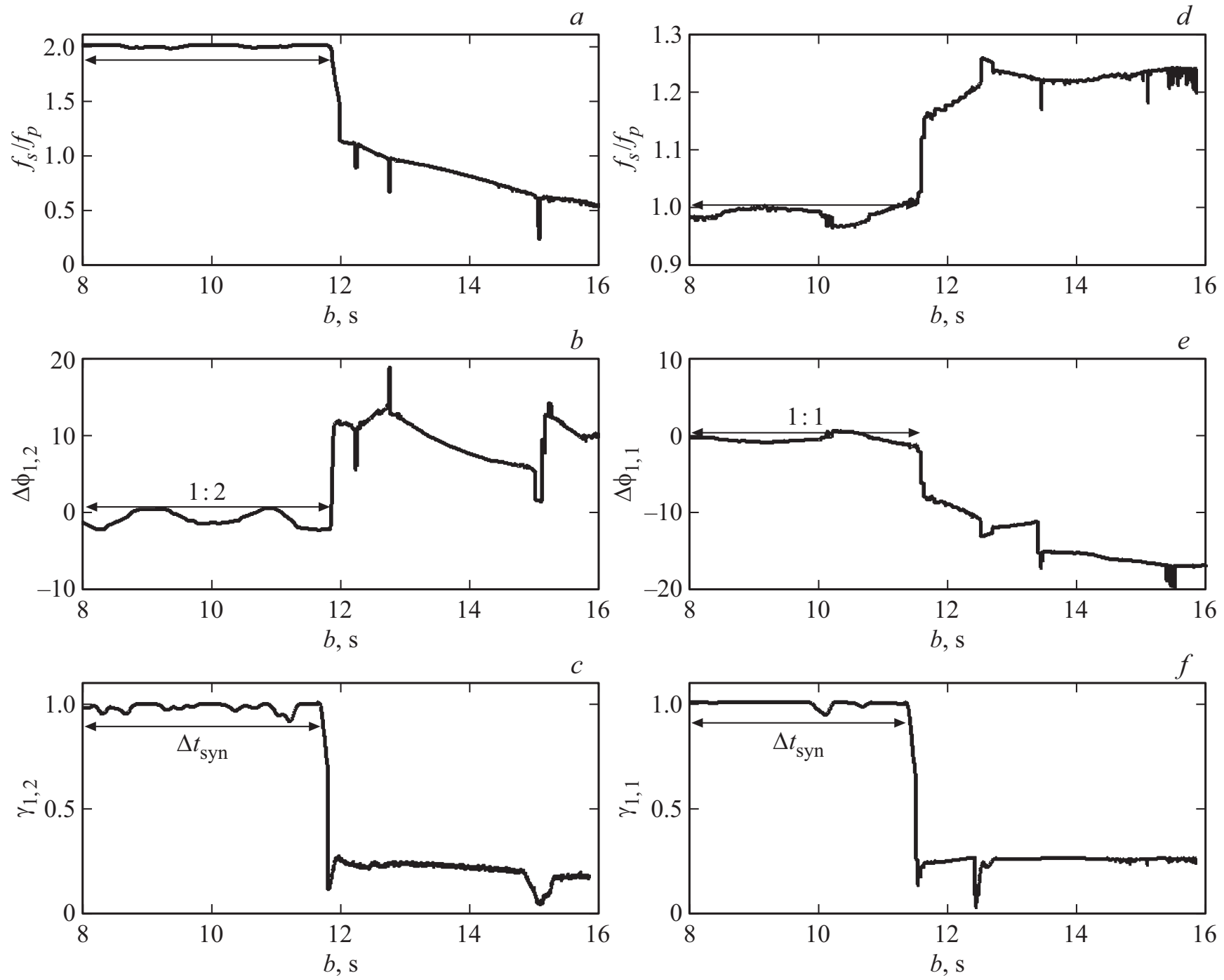

Рис. 6. Фазовая синхронизация порядков $1: 2$ и $1: 1$ между световым возбуждением с частотой 4 Нz и ответом мозга человека из группы II. $a, d-$ отношение мгновенных частот $f_{s}(b) / f_{p}(b), b, e-$ мгновенные разности фаз $\Delta \phi_{1,2}(b)$ и $\Delta \phi_{1,1}(b), c, f-$ индексы фазовой синхронизации $\gamma_{1,2}$ и $\gamma_{1,1}$.

В паттернах ЭЭГ 100\% (10 из 10) лиц из контрольной группы отмечалась фазовая синхронизация порядка $1: 1$ только между световым возбуждением с частотой $12 \mathrm{~Hz}$ и биоэлектрической активностью мозга (табл. 3). Для других частот возбуждения фазовая синхронизация отсутствовала. Отсутствие фазовой синхронизации между ответом мозга и фотостимулом большинства предложенных частот совпадает с ранее полученными данными о том, что реактивные паттерны ЭЭГ здорового человека характеризуются незначительной реакцией усвоения ритма фотостимуляции частот альфа- диапазона $[3,8]$.

Таким образом, в отличие от биоэлектрической активности здорового мозга, в паттернах ЭЭГ обеих групп пациентов с артериальной гипертензией наблюдалась реакция усвоения частот тета- и альфа- диапазонов, связанная с фазовой синхронизацией порядка $1: 1$, при которой вызванный ответ имел ту же частоту, что и стимул. Наблюдаемая в паттернах ЭЭГ пациентов из группы I фазовая синхронизация порядков $2: 1,3: 2$, $3: 1,4: 1$ означает возникновение частоты ответа, кратно меньшей по сравнению с частотой возбуждения, например, появление в паттерне ЭЭГ частоты $6 \mathrm{~Hz}$ в ответ на фотостимул с частотой $18 \mathrm{~Hz}$ или частоты $5 \mathrm{~Hz}$ в ответ на стимул $10 \mathrm{~Hz}$. Отношение мгновенных частот $f_{s}(b) / f_{p}(b)$ имеет в этих случаях значение, меньшее единицы (табл. 3).

В отличие от этого характерная для пациентов из группы II фазовая синхронизация порядка 1:2 означает появление в паттерне ЭЭГ частоты, кратно большей, по сравнению с частотой возбуждения, например, возникновение ответа с частотой $8 \mathrm{~Hz}$ на фотостимул с частотой $4 \mathrm{~Hz}$ или ответа с частотой $12 \mathrm{~Hz}$ на световое возбуждение с частотой $6 \mathrm{~Hz}$. Отношение мгновенных 
частот $f_{s}(b) / f_{p}(b)$ имеет в этих случаях значение большее единицы (табл. 3 ).

Попарное сравнение длительностей фазовой синхронизации порядка 1:1 между группами I и II и между группой II и контрольной группой, выполненное на основании однофакторного дисперсионного анализа, представлено на рис. 7. Средние величины $\Delta t_{\mathrm{syn}}$ определены по восьми значениям для каждой анализируемой группы. В связи с отсутствием данных о наличии фазовой синхронизации одного и того же порядка сразу для трех анализируемых групп, тест множественных сравнений не проводился. Рис. 7 демонстрирует достоверные различия между средними значениями длительностей фазовой синхронизации порядка $1: 1$ для частот возбуждения 4, 6 и $10 \mathrm{~Hz}$ в паттернах ЭЭГ групп I и II пациентов с артериальной гипертензией, а также для частоты возбуждения $12 \mathrm{~Hz}$ в паттернах ЭЭГ контрольной группы и группы II. Большие различия в центральных линиях, показывающих значения медиан выборочных значений, соответствуют большим значениям статистики Фишера и соответственно малым значениям $p(p<0.002)$. Это свидетельствует о том, что групповые средние длительностей фазовой синхронизации порядка $1: 1$ различаются на уровне значимости $\alpha<0.05$. При этом для всех рассмотренных частот длительность фазовой синхронизации порядка 1:1 в паттернах ЭЭГ пациентов из группы I практически в 2 раза превышает длительность синхронизации для пациентов из группы II (например, при $f_{P}=4 \mathrm{~Hz} \quad \Delta t_{\mathrm{syn}}=6.3 \pm 0.9 \mathrm{~s} \quad$ и $\Delta t_{\mathrm{syn}}=3.8 \pm 0.4 \mathrm{~s}$

Таблица 1. Порядки $n: m$ фазовой синхронизации между фотостимулом и реакцией мозга пациентов с артериальной гипертензией из группы I

\begin{tabular}{c|c|c|c|c|c|c}
\hline \multirow{2}{*}{ Пациент } & \multicolumn{6}{|c}{ Синхронизия $n: m$} \\
\cline { 2 - 7 } & $4 \mathrm{~Hz}$ & $6 \mathrm{~Hz}$ & $10 \mathrm{~Hz}$ & $12 \mathrm{~Hz}$ & $16 \mathrm{~Hz}$ & $18 \mathrm{~Hz}$ \\
\hline 1 & $1: 1$ & $1: 1$ & $1: 1,2: 1$ & $2: 1,3: 2$ & $4: 1$ & $3: 1$ \\
\hline 2 & - & $1: 1$ & $1: 1$ & $2: 1,3: 1$ & $4: 1$ & - \\
\hline 3 & $1: 1$ & $1: 1$ & $1: 1,2: 1$ & $2: 1,3: 2$ & $4: 1$ & $3: 1$ \\
\hline 4 & $1: 1$ & $1: 1$ & $2: 1$ & $2: 1$ & - & $3: 1$ \\
\hline 5 & $1: 1$ & - & $2: 1$ & $2: 1,3: 2$ & $4: 1$ & - \\
\hline 6 & $1: 1$ & $1: 1$ & $1: 1$ & $3: 2$ & - & $3: 1$ \\
\hline 7 & - & $1: 1$ & $2: 1$ & $2: 1$ & $4: 1$ & $3: 1$ \\
\hline 8 & $1: 1$ & $1: 1$ & $2: 1$ & $2: 1,3: 2$ & - & $3: 1$ \\
\hline 9 & $1: 1$ & $1: 1$ & $1: 1,2: 1$ & - & $4: 1$ & - \\
\hline 10 & - & $1: 1$ & $1: 1,2: 1$ & $2: 1,3: 2$ & $4: 1$ & $3: 1$ \\
\hline 11 & $1: 1$ & - & $1: 1,2: 1$ & $2: 1,3: 2$ & $4: 1$ & - \\
\hline 12 & $1: 1$ & $1: 1$ & - & $2: 1,3: 1$ & $4: 1$ & $3: 1$ \\
\hline 13 & $1: 1$ & - & $1: 1$ & $2: 1,3: 2$ & $4: 1$ & - \\
\hline & & & & & & \\
\hline
\end{tabular}

Таблица 2. Порядки $n: m$ фазовой синхронизации между фотостимулом и реакцией мозга пациентов с артериальной гипертензией из группы II

\begin{tabular}{|c|c|c|c|c|}
\hline \multirow{2}{*}{ Пациент } & \multicolumn{4}{|c|}{ Синхронизация $n: m$} \\
\hline & $4 \mathrm{~Hz}$ & $6 \mathrm{~Hz}$ & $10 \mathrm{~Hz}$ & $12 \mathrm{~Hz}$ \\
\hline 1 & $1: 1,4: 5,4: 7,1: 2$ & $1: 1,1: 2$ & $1: 1$ & $1: 1$ \\
\hline 2 & $1: 1,1: 2$ & $1: 1$ & $1: 1$ & $1: 1$ \\
\hline 3 & $1: 1$ & $1: 1$ & $1: 1$ & - \\
\hline 4 & $1: 1$ & $1: 1,1: 2$ & $1: 1$ & $1: 1$ \\
\hline 5 & $1: 1,1: 2$ & $1: 1,1: 2$ & $1: 1$ & - \\
\hline 6 & $1: 1,1: 2$ & $1: 1,1: 2$ & $1: 1$ & $1: 1$ \\
\hline 7 & $1: 1,1: 2$ & $1: 1$ & $1: 1$ & $1: 1$ \\
\hline 8 & $1: 1,1: 2$ & $1: 1,1: 2,1: 3$ & $1: 1$ & $1: 1$ \\
\hline 9 & $1: 1$ & $1: 1,1: 2$ & $1: 1$ & - \\
\hline 10 & $1: 1$ & $1: 1$ & $1: 1$ & $1: 1$ \\
\hline 11 & $1: 1,1: 2$ & $1: 1,1: 2$ & $1: 1$ & - \\
\hline 12 & $1: 1,1: 2$ & $1: 1,1: 2$ & $1: 1$ & $1: 1$ \\
\hline 13 & $1: 1,1: 2$ & $1: 1,1: 2$ & $1: 1$ & $1: 1$ \\
\hline 14 & - & $1: 1,1: 2,1: 3$ & $1: 1$ & - \\
\hline 15 & $1: 1,1: 2$ & $1: 1,1: 2,1: 3$ & - & - \\
\hline 16 & $1: 1,1: 2$ & $1: 1,1: 2,1: 3$ & - & $1: 1$ \\
\hline 17 & $1: 1,1: 2$ & $1: 1$ & $1: 1$ & - \\
\hline
\end{tabular}

для группы I и II соответственно). Наименьшие значения длительности фазовой синхронизации получены в ответах здоровых тестируемых $\left(\Delta t_{\mathrm{syn}}=1.7 \pm 0.3 \mathrm{~s}\right.$, $\left.f_{P}=12 \mathrm{~Hz}\right)$.

Таким образом, в отличие от реакции мозга здоровых людей из контрольной группы, у которых реакция усвоения светового ритма наблюдается только для определенных частот альфа-диапазона, в паттернах ЭЭГ пациентов с артериальной гипертензией реакция усвоения светового ритма сопровождается появлением фазовой синхронизации в ответах мозга практически во всех частотных диапазонах. В реактивных паттернах ЭЭГ пациентов с умеренными когнитивными нарушениями, вызванными хроническим повышением артериального давления (группа I), повышается длительность фазовой синхронизации порядка 1:1 при световой стимуляции в тета- и альфа-диапазонах. Кроме того, при стимуляции на частотах альфа- и бета-диапазонов реакции мозга пациентов с умеренными когнитивными нарушениями смещаются в более низкочастотный диапазон по сравнению с частотой возбуждения. Фазовая синхронизация порядка $m: n$, где $m>n$, приводящая к уменышению частоты ответа мозга по сравнению с 
Таблица 3. Усредненные по тестируемым в каждой группе значения отношения мгновенных частот $f_{s}(b) / f_{p}(b)$ во время фазовой синхронизации порядка $n: m$

\begin{tabular}{|c|c|c|c|c|c|c|}
\hline \multirow{2}{*}{$\frac{\text { Фотостимул }}{f_{P}(\mathrm{~Hz})}$} & \multicolumn{2}{|c|}{ Контрольная группа $(N=10 / 10)$} & \multicolumn{2}{|c|}{ Группа I $(N=8 / 13)$} & \multicolumn{2}{|c|}{ Группа II $(N=12 / 17)$} \\
\hline & $f_{s}(b) / f_{p}(b)$ & $n: m$ & $f_{s}(b) / f_{p}(b)$ & $n: m$ & $f_{s}(b) / f_{p}(b)$ & $n: m$ \\
\hline \multirow{2}{*}{4} & \multirow{2}{*}{-} & \multirow{2}{*}{-} & \multirow{2}{*}{$1 \pm 0.01$} & \multirow{2}{*}{$1: 1$} & $1 \pm 0.01$ & $1: 1$ \\
\hline & & & & & $2 \pm 0.03$ & $1: 2$ \\
\hline \multirow{2}{*}{6} & \multirow{2}{*}{-} & \multirow{2}{*}{-} & \multirow{2}{*}{$1 \pm 0.01$} & \multirow{2}{*}{$1: 1$} & $1 \pm 0.01$ & $1: 1$ \\
\hline & & & & & $2 \pm 0.02$ & $1: 2$ \\
\hline \multirow{2}{*}{10} & \multirow{2}{*}{-} & \multirow{2}{*}{-} & $1 \pm 0.01$ & $1: 1$ & \multirow{2}{*}{$1 \pm 0.01$} & \multirow{2}{*}{$1: 1$} \\
\hline & & & $0.5 \pm 0.01$ & $2: 1$ & & \\
\hline \multirow{2}{*}{12} & \multirow{2}{*}{$1 \pm 0.01$} & \multirow{2}{*}{$1: 1$} & $0.5 \pm 0.01$ & $2: 1$ & \multirow{2}{*}{$1 \pm 0.01$} & \multirow{2}{*}{$1: 1$} \\
\hline & & & $0.66 \pm 0.01$ & $3: 2$ & & \\
\hline 16 & - & - & $0.25 \pm 0.01$ & $4: 1$ & - & - \\
\hline 18 & & & $0.33 \pm 0.01$ & $3: 1$ & - & - \\
\hline
\end{tabular}

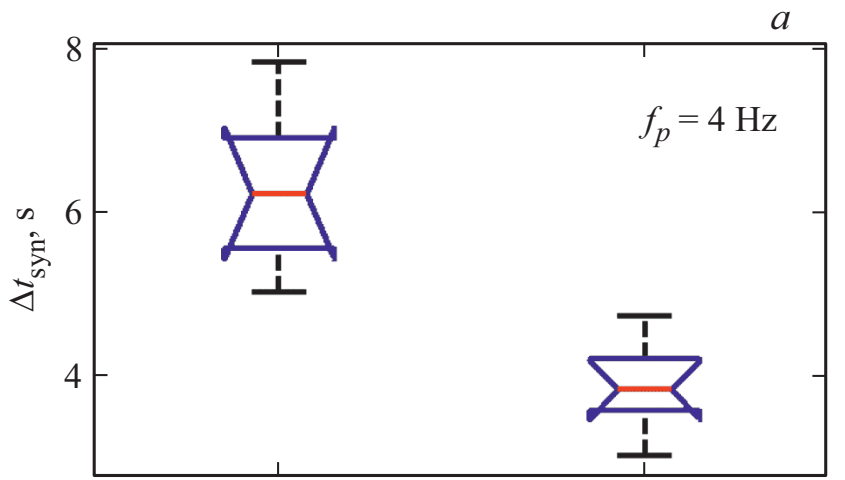

Group I

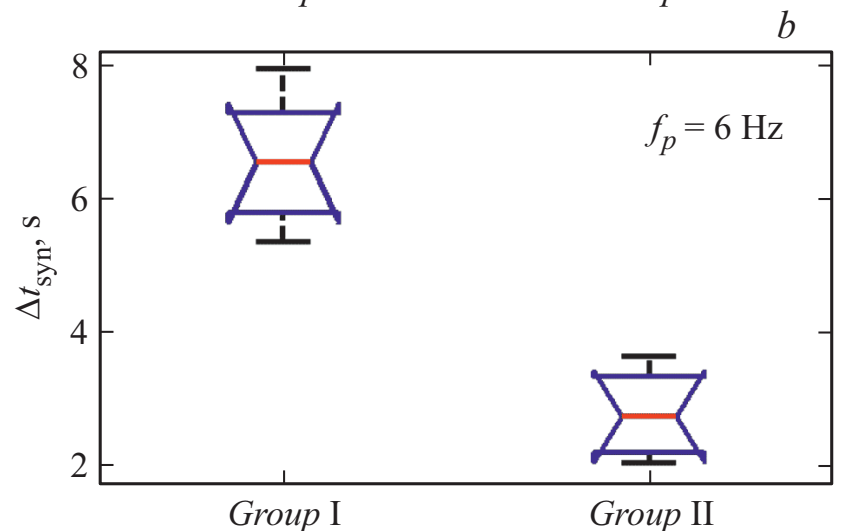

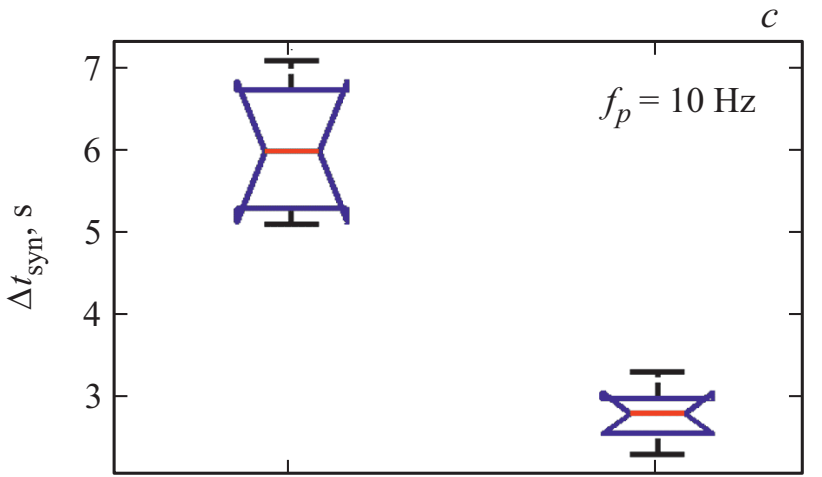

Group I

Group II

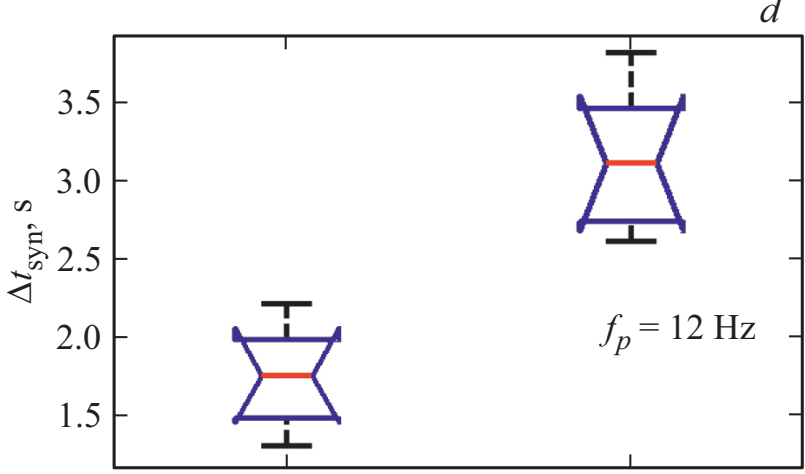

Control

Group II

Рис. 7. Сравнение длительностей фазовой синхронизации порядка $1: 1$ между группами I и II для частот возбуждения 4, 6, $10 \mathrm{~Hz}(a-c)$ и между группой II и контрольной группой для частоты фотостимула $12 \mathrm{~Hz}(d)$.

частотой светового раздражителя, а также увеличение времени фазовой синхронизации, могут быть маркерами умеренных когнитивных нарушений при артериальной гипертензии.

\section{Выводы}

Анализ фазовой синхронизации между паттернами ЭЭГ и фотостимулом, выполненный с помощью син- 
хросжатого вейвлет-преобразования, позволил выявить достоверные различия в параметрах фазовой синхронизации в двух группах лиц с хронически повышенным артериальным давлением, имеющих и не имеющих умеренные нарушения когнитивных функций.

Показано, что умеренные когнитивные нарушения у лиц с хроническим повышением артериального давления коррелируют с большей длительностью фазовой синхронизации между прерывистой фотостимуляцией и биоэлектрической активностью головного мозга, а также со сдвигом этой активности в более низкочастотный диапазон по сравнению с частотой возбуждения. Это позволяет считать, что параметры фазовой синхронизации в электроэнцефалографических паттернах могут служить дополнением к клиническим оценкам степени умеренных когнитивных нарушений сосудистого генеза и быть нейрофизиологическими маркерами этих нарушений.

\section{Благодарности}

Авторы благодарят старшего научного сотрудника Института физиологии им. И.П. Павлова Святогор И.А. за предоставленные экспериментальные записи ЭЭГ.

\section{Конфликт интересов}

Авторы заявляют, что у них нет конфликта интересов.

\section{Список литературы}

[1] J.M.E. Drake., K.E. Shy, L. Liss. Clin. Electroencephal, 20, 153 (1989).

[2] A.I. Fedotchev, A.T. Bondar, S.G. Matrusov, V.S. Semenov, A.G. Soin. Usp. Physiol Nauk, 37 (4), 82 (2006).

[3] O.E. Dick, I.A. Svyatogor. Neurocomputing, 165, 361 (2015). http://dx.doi.org/10.1016/j.neucom.2015.03.025

[4] A.I. Fedotchev, A.T. Bondar, I.G. Akoev. Human Physiology, 26, 64 (2000).

[5] M. Coull, T.A. Pedley. Electroenceph. Clin. Neurophys, 44, 353 (1978).

[6] D. Gasecki, M. Kwarciany, W. Nyka, K. Narkiewicz. Curr Hypertens Rep., 15 (6), 547 (2013). DOI: $10.1007 / \mathrm{s} 11906-013-0398-4$

[7] D.V. Moretti, C. Miniussi, G.B. Frison. Clin. Neurophys., 118, 1866 (2007).

[8] И.А. Святогор, О.Е. Дик, А.Д. Ноздрачев, Н.Л. Гусева. Физиология человека, $\mathbf{4 1}(3), 41$ (2015). DOI: 10.7868/S0131164615030170 [O.E. Dick, I.A. Svyatogor, A.D. Nozdrachev, N.L. Guseva. Human Physiology, 41, 261 (2015). DOI: $10.1134 / \mathrm{S} 0362119715030172]$

[9] С.В. Божокин. ЖТФ, 80, 16 (2010). [S.V. Bozhokin, Tech. Phys. 55, 1248 (2010). doi.org/10.1134/S1063784210090033]

[10] S. Boccaletti, J. Kurths, G. Osipov, D.L. Valladares, C.S. Zhou. Physics Reports, 366 (1-2), 1 (2002).

DOI: $10.1016 / \mathrm{S} 0370-1573(02) 00137-0$
[11] A.S. Pikovsky, M.G. Rosenblum, J. Kurths. Synchronization: a universal concept in nonlinear sciences (Cambridge Univer. Press, Cambridge, 2001)

[12] L. Cohen. Time-frequency analysis (Prentice Hall Signal Processing Series, Prentice Hall, Englewood Cliffs, New Jersey, 1995)

[13] B. Kralemann, M. Fruhwirth, A. Pikovsky, M. Rosenblum, T. Kenner, J. Schaefer, M. Moser. Nature Communications, 4, 2418 (2013). DOI: $10.1038 /$ ncomms 3418

[14] M.G. Rosenblum, L. Cimponeriu, A. Bezerianos, A. Patzak, R. Mrowka. Phys. Rev. E, 65, 041909-1 (2002). DOI: 10.1103/PhysRevE.65.041909

[15] Q. Zhang, A.R. Patwardhan, C.F. Knapp, J.M. Evans. Eur. J. Appl. Physiol., 115 (2), 417 (2015). DOI: $10.1007 / \mathrm{s} 00421-014-3017-4$

[16] I. Daubechies. Ten lectures on Wavelets (Mathematics, SIAM Publication, Philadelphia, Pennsylvania, 1992)

[17] A.B. Bespyatov, M.B. Bodrov, V.I. Gridnev, V.I. Ponomarenko, M.D. Prokhorov. Nonlin. Phen. Compl. Syst., 6 (4), 885 (2003).

[18] E. Hramov, A.A. Koronovskii, V.I. Ponomarenko, M.D. Prokhorov. Phys. Rev. E, 73, 026208-1 (2006). DOI: 10.1103/PhysRevE.73.026208

[19] O.E. Dick, I.A. Svyatogor. Neurocomputing, 82, 207 (2012). DOI: $10.1016 /$ j.neucom.2011.11.013

[20] O.E. Dick. Neurocomputing, 243, 142 (2017). http://dx.doi.org/10.1016/j.neucom.2017.03.008

[21] I. Daubechies, J. Lu, H.T. Wu. Appl. Comput. Harmon. Anal., 30, 243 (2011). DOI: 10.1016/j.acha.2010.08.002

[22] J.B. Tary, R.H. Herrera, M. van der Baan. Phil. Trans. R. Soc. A, 376, 2126 (2018). https://dx.doi.org/10.6084/m9.

[23] D. Iatsenko, P.V.E. McClintock, A. Stefanovska. Digital Signal Proc., 42, 1 (2015). http://dx.doi.org/10.1016/j.dsp.2015.03.004

[24] G. Thakur, E. Brevdo, N.S. Fuckar, H.-T. Wu. Signal Proc., 93, 1079 (2013).

[25] G.P. Torrence, Bull. Am. Meteorol. Soc., 79, 61 (1998).

[26] R.Q. Quiroga, A. Kraskov, T. Kreuz, P. Grassberger. Phys.Rev. E, 65, 041903 (2002). DOI: 10.1103/PhysRevE.65.041903

[27] F. Mormann, K. Lehnertz, P. David, C.E. Elger. Phys. D, 144, 358 (2000). 